\section{CONTINUOUS TRACHEAL INTUBATION IN PATIENTS WITH MYASTHENIA GRAVIS AFTER THYMECTOMY TO PREVENT TRACHEAL INCISION FOR MYASTHENIC CRISIS}

\section{To the Editor:}

We read the article by Watanabe and colleagues ${ }^{1}$ with great interest. In the article the authors analyzed 122 patients who underwent transsternal thymectomy and found that myasthenic crisis was affected by the existence of preoperative bulbar symptoms, a history of preoperative myasthenic crisis, a preoperative serum level of antiacetylcholine receptor antibody of greater than $100 \mathrm{nmol} / \mathrm{L}$, and intraoperative blood loss of greater than $1000 \mathrm{~mL}$. They concluded that meticulous preoperative and postoperative care should be carried out to prevent postoperative myasthenic crisis in patients with these prognostic factors.

Herein we present tips on continuous tracheal intubation in patients with myasthenia gravis after thymectomy to prevent tracheal incision for myasthenic crisis. We separated 236 patients with myasthenia gravis who had undergone thymectomy by means of video-assisted thoracoscopic surgery into 2 groups. We prospectively continued tracheal intubation to 48 hours in group A (120 patients). The other 116 patients in group B refused the procedures and were regarded as negative control subjects. We found that the strong predictors of myasthenia crisis included pulmonary complications, Osserman typing of IIb or greater, malignant thymoma, and a preoperative oral dose of pyridostigmine bromide of greater than $480 \mathrm{mg} / \mathrm{d}$. There were not any differences regarding the incidence rate of myasthenia crisis between groups A and B. However, the requirement of tracheal incision in group A was much lower than in group B $(10 \%$ vs $29.3 \%, P<.01)$ because most of the crisis happened postoperatively in 48 hours and continuous intubation could support many patients until attenuation of the symptoms.

As a result, we think that postoperative continuous tracheal intubation to 48 hours is necessary for those patients with myasthenia gravis who are prone to crisis.

Bo Deng, $M D$

Zheng $M a, M D$

Yao-Guang Jiang, $M D$

Ru-Wen Wang, $M D$

Qun-You Tan, $M D$

Yun-Ping Zhao, $M D$

Jing-Hai Zhou, MD

Tai-Qian Gong, MD

Thoracic Surgery Department Institute of Surgery Research

Daping Hospital

Third Military Medical University

Chongqing City, P.R. China.

\section{Reference}

1. Watanabe A, Watanabe T, Obama T, Mawatari T, Ohsawa H, Ichimiya Y, et al. Prognostic factors for myasthenic crisis after transsternal thymectomy in patients with myasthenia gravis. J Thorac Cardiovasc Surg. 2004;127:868-76.

\section{doi:10.1016/j.jtcvs.2011.02.041}

\section{Reply to the Editor:}

We would like to congratulate Deng and colleagues on their letter with comments on our article, ${ }^{1}$ especially on the comparison of the outcomes between the 2 studies. First of all, we have to understand that the surgical approach of our study has major differences from their study. In our study extended thymectomy through a median sternotomy was performed, whereas thymectomy by means of video-assisted thoracoscopic surgery was their technique. The incidence of tracheostomy was $1.6 \%(2 / 122)$ for our study compared with $10 \%$ in the intervention group for their study. We attributed the difference in indication for tracheostomy to the different incidence results. Tracheal intubation or noninvasive ventilation ${ }^{2}$ was maintained in our patients for at least 7 days when no inherent pulmonary disease was noted. Furthermore, blood absorption was actively used for patients with a preoperative risk of postoperative myasthenic crisis or those who experienced a postoperative myasthenic crisis.

Second, they described the tips on continuous tracheal intubation (for 48 hours) in patients with myasthenia gravis after thymectomy to prevent tracheal incision for myasthenic crisis. In their study most of the postoperative myasthenic crisis occurred within 2 days after thymectomy; however, in our study it occurred within an average of 5 days (range, 1-17 days) after thymectomy. In addition, the rate of postoperative myasthenic crisis within 2 days after thymectomy was only $35.7 \%$ of patients with postoperative myasthenic crisis. Therefore in our study most patients could have adverse effects from the use of continuous tracheal intubation. We preferred early extubation ratherthan continuous intubation to prevent pulmonary complications.

Third, Deng and colleagues revealed that the strong predictors of myasthenic crisis included pulmonary complications, Osserman typing of IIb or greater, malignant thymoma, and preoperative oral dose of pyridostigmine bromide of greater than 480 $\mathrm{mg} / \mathrm{d}$. Only Osserman typing of IIb or greater (ie, bulbar symptom) was the common predictor between the 2 studies. The differences were a result of the differences in surgical approach, perioperative management, and so on. Recently, our group performed thymectomy with videoassisted thoracoscopic surgery on 17 patients with myasthenia gravis, and none of them experienced postoperative myasthenic crisis.

Finally, it is important to know the risks of postoperative myasthenic crisis in accordance with the situation, such as surgical approach, perioperative management, and patient selection.

Atsushi Watanabe, MD, PhD

Department of Thoracic and Cardiovascular Surgery

Sapporo Medical University School of Medicine

Sapporo, Japan 


\section{References}

1. Watanabe A, Watanabe T, Obama T, Mawatari T, Ohsawa H, Ichimiya Y, et al. Prognostic factors for myasthenic crisis after transsternal thymectomy in patients with myasthenia gravis. J Thorac Cardiovasc Surg. 2004;127:868-76.

2. Wu JY, Kuo PH, Fan PC, Wu HD, Shih FY Yang PC. The role of non-invasive ventilation and factors predicting extubation outcome in myasthenic crisis. Neurocrit Care. 2009;10:35-42.

doi:10.1016/j.jtcvs.2011.03.023
THE GORDIAN KNOT OF REVASCULARIZATION FOR MULTIVESSEL CORONARY ARTERY DISEASE

To the Editor:

We read with great interest a recent meta-analysis by Yan and associates ${ }^{1}$ of randomized and norandomized studies of percutaneous coronary intervention with drug-eluting stents (DESs) versus coronary artery bypass grafting (CABG) for coronary artery disease, including not only multivessel disease (MVD) but also single-vessel disease or left main coronary artery disease. This meta-analysis, ${ }^{1}$ as well as a previous meta-analysis of MVD, ${ }^{2}$ demonstrated no difference in all-cause mortality at least 1 year after DES and CABG treatments. Although these

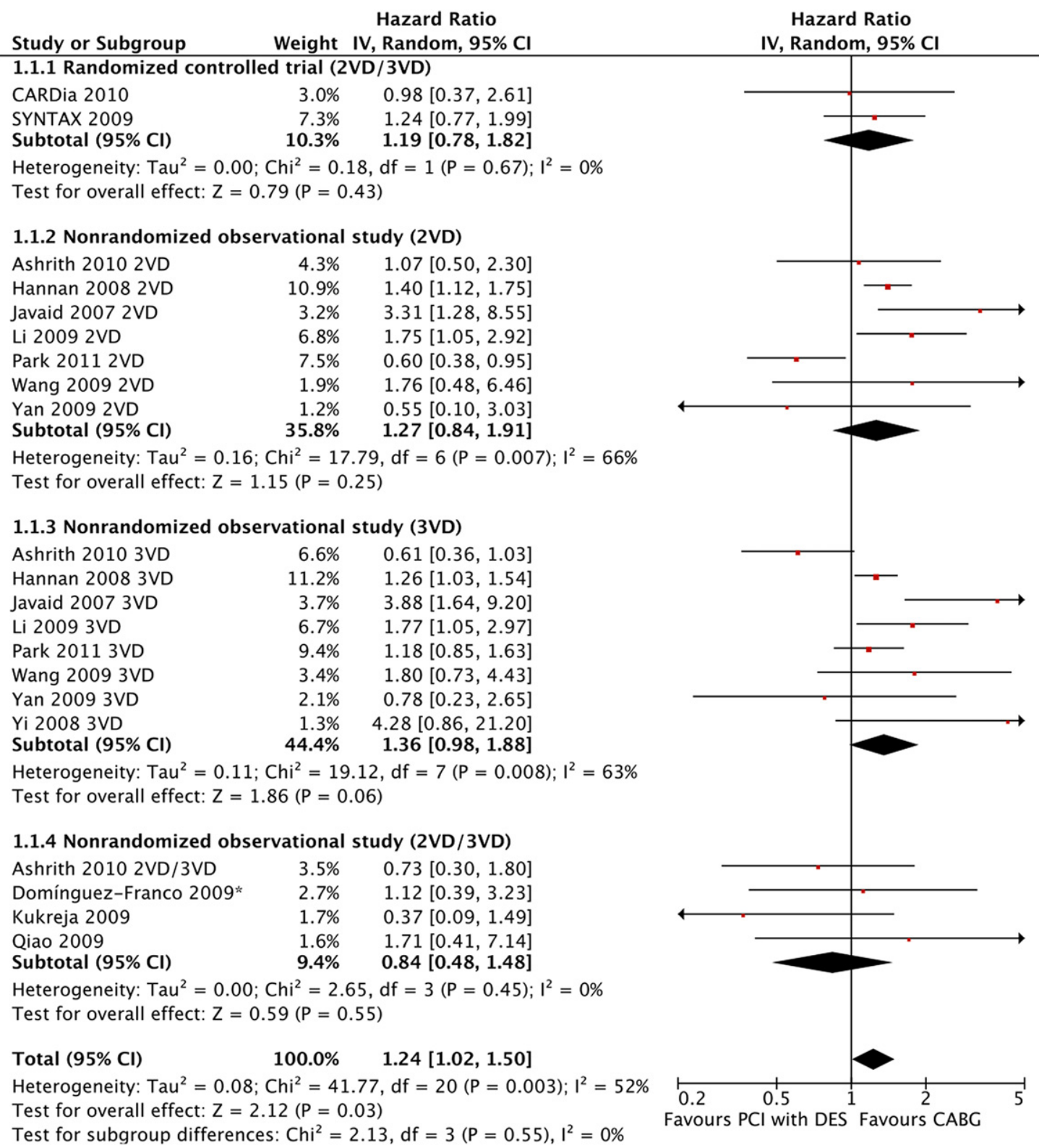

FIGURE 1. Follow-up ( $\geq 1$ year) all-cause mortality among patients with multivessel disease assigned to percutaneous coronary intervention $(P C I)$ with drug-eluting stent $(D E S)$ versus coronary artery bypass grafting $(C A B G)$. Asterisk indicates that the study provided an adjusted odds ratio. $I V$, Inverse variance; $C I$, confidence interval; $2 V D$, double-vessel disease; $3 V D$, triple-vessel disease; CARDia, Coronary Artery Revascularization in Diabetes trial ${ }^{4}$; SYNTAX, Synergy between Percutaneous Coronary Intervention with Taxus and Cardiac Surgery trial. ${ }^{5}$ 